\title{
PROJECTIVE REPRESENTATIONS AND INDUCED LINEAR CHARACTERS ${ }^{1}$
}

T. V. FOSSUM

0. Introduction and notation. The purpose of this paper is to generalize arithmetic results obtained by $\mathrm{C}$. W. Curtis and the author in [1] concerning the degrees of irreducible representations of finite groups. Although the generalization given in $\$ 2$ is somewhat crude because of the multitude of hypotheses, the necessary groundwork devoted to projective representations in $\$ 1$ seems to be of interest in itself.

All groups considered here are finite. Let $G$ be a group, $H$ a subgroup of $G$, and suppose $x \in G$. We will adhere to the following notations throughout this paper:

$$
\begin{gathered}
|G|=\text { order of } G, \quad h^{x}=x^{-1} h x \quad(h \in H), \\
H^{x}=\left\{h^{x}: h \in H\right\}, \quad H^{(x)}=H \cap H^{x}, \quad \operatorname{ind}_{I I} x=\left[H: H^{(x)}\right] .
\end{gathered}
$$

The symbol $K$ will always stand for the complex field, and all characters are assumed to be $K$-characters. If $\zeta$ is a character of $H$, define $\zeta^{x}: H^{x} \rightarrow K$ by $\zeta^{x}\left(h^{x}\right)=\zeta(h),\left(h^{x} \in H^{x}\right)$. Then $\zeta^{x}$ is a $K$-character of $H^{x}$. All other notations are standard and are included in [2].

As a foundation for our results we state the following theorem, the proof of which is the subject of [1].

Theorem A. Let $H$ be a subgroup of the group G. Suppose $\zeta$ is a linear character of $H$ and $\chi$ an irreducible character of $G$ such that $\left(\zeta^{G}, \chi\right)>0$. Set $e=|H|^{-1} \sum_{h \in H} \zeta\left(h^{-1}\right) h$, the primitive idempotent in $K H$ such that $K H e$ affords $\zeta$. Then the restriction $\chi_{E}$ of $\chi$ to $E=e K G e$ is an irreducible character of $E$. If $\left\{g_{i}\right\}$ is a set of representatives of the distinct $(H, H)$ double cosets $H \chi H$ for which $\zeta^{x}=\zeta$ on $H^{(x)}$, then the set of $a_{i}=\left(\operatorname{ind}_{H} g_{i}\right) e g_{i} e$ is a basis for $E=e K G e$. Moreover if $b_{i}=\left(\operatorname{ind}_{H} g_{i}\right) e g_{i}^{-1} e$, then the block idempotent of $E$ corresponding to $\chi_{E}$ is

$$
(\chi(1) /[G: H]) \sum_{i}\left(\operatorname{ind}_{H} g_{i}\right)^{-1} \chi\left(b_{i}\right) a_{i} \text {. }
$$

Finally, the degree $\chi(1)$ of $\chi$ divides

$$
[G: H] \operatorname{lcm}\left\{\operatorname{ind}_{H} g_{i}\right\}=[G: H] \operatorname{lcm}\left\{\operatorname{ind}_{H} x: \zeta^{x}=\zeta \text { on } H^{(x)}, x \in G\right] \text {. }
$$

Received by the editors May 26, 1969.

1 This paper is a portion of a Ph.D. thesis by the author submitted to the University of Oregon, August, 1968. 
1. Characters and twisted group-algebras. Assume $S$ is a group, $f: S \times S \rightarrow K^{*}=K \backslash\{0\}$ a factor set of $S$ whose values are powers of a fixed primitive $n$th root of unity $w$ in $K$. (Every factor set can be so normalized. See $[2, \S 53]$.) Let $K(S)_{f}$ be the (associative) twisted group-algebra with $K$-basis $\{[s]: s \in S\}$ in one-to-one correspondence with the elements of $S$ and with multiplication given by $[g][h]$ $=f(g, h)[g h], \quad(g, h \in S)$. Finally assume [1] is the multiplicative identity for $K(S)_{f}$.

Let $S_{f}$ denote the finite multiplicative subgroup of the group of units of $K(S)_{f}$ consisting of elements of the form $w^{i}[s], 0 \leqq i \leqq n-1$, $s \in S$. Then [1] is the identity of $S_{f}$ and $S_{f}$ contains a cyclic central subgroup $C_{f}=\left\{w^{i}[1]: 0 \leqq i \leqq n-1\right\}$ such that $S_{f} / C_{f} \simeq S$. If $K S_{f}$ denotes the ordinary group-algebra of $S_{f}$ over $K$ then the map $\Phi: K S_{f}$ $\rightarrow K(S)_{f}$ given by $\Phi\left(w^{i}[s]\right)=w^{i} \cdot[s]$ (extended linearly to $K S_{f}$ ) is a $K$-algebra epimorphism. The element

$$
e_{f}=1 / n \sum_{i=0}^{n-1} w^{-i}\left(w^{i}[1]\right)
$$

is an idempotent in $K C_{f}$ (see $[2, \S 33]$ ) which is central in $K S_{f}$ such that $\Phi\left(e_{f}\right)=[1]$, and $e_{f}\left(K S_{f}\right) e_{f} \simeq K(S)_{f}$ under $\Phi$.

Now suppose $T$ is a subgroup of $S$ and construct the unital subalgebra $K(T)_{f}$ of $K(S)_{f}$ with the factor set $f$ restricted to $T \times T$. Similar to $S_{f}$ construct $T_{f}=\left\{w^{i}[t]\right\}$ with cyclic central subgroup $C_{f}$ such that $T_{f} / C_{f} \simeq T$. Then the restriction $\Phi^{\prime}$ of $\Phi$ to $K T_{f}$ is an algebra epimorphism of $K T_{f}$ onto $K(T)_{f}$.

Suppose $\chi$ is an irreducible character of $K(S)_{f}$. We pull back $\chi$ to $K S_{f}$ by defining $\chi_{f}=\chi \Phi$. Then $\chi_{f}$ is an irreducible character of $K S_{f}$ of degree $\chi_{f}([1])=\chi([1])$ and its corresponding block idempotent in $K S_{f}$ is

$$
\epsilon=\chi_{f}([1])\left|S_{f}\right|^{-1} \sum_{o \in S_{f}} \chi_{f}\left(g^{-1}\right) g
$$

(see $[2, \S 33])$. By definition of $\Phi$,

$$
\Phi(\epsilon)=\chi([1])|S|^{-1} \sum_{s \in S} \chi\left([s]^{-1}\right)[s],
$$

since $\left|S_{f}\right|=n|S|$ and $\chi([1])=\chi_{f}([1])$. Moreover $\Phi(\epsilon)$ is the block idempotent in $K(S)_{f}$ corresponding to $\chi$.

Suppose further that $\zeta: K(T)_{f} \rightarrow K$ is an algebra homomorphism. ${ }^{2}$ Then we pull back $\zeta$ to $\zeta_{f}=\zeta \Phi^{\prime}$, which is clearly an algebra homomorphism of $K T_{f}$ onto $K$. If $\chi$ restricted to $K(T)_{f}$ contains $\zeta$ (i.e., if

\footnotetext{
${ }^{2}$ Since $\zeta$ is linear on $K(T)_{f}, f$ is a trivial cocycle on $T$ and therefore $K(T)_{f} \cong K T$.
} 
the module affording $\chi$, restricted to $K(T)_{f}$, has the module affording $\zeta$ as a composition factor) then $\chi_{f}$, restricted to $K T_{f}$, contains $\zeta_{f}$. By Theorem A above, the degree $\chi_{f}([1])$ of $\chi_{f}$ divides

$$
\left[S_{f}: T_{f}\right] \operatorname{lcm}\left\{\operatorname{ind}_{T_{f}} g: \zeta_{f}^{0}=\zeta_{f} \text { on } T_{f}^{(o)}, g \in S_{f}\right\} .
$$

First note that $\left[S_{f}: T_{f}\right]=[S: T]$. Moreover one checks that

$$
\begin{aligned}
& \operatorname{lcm}\left\{\operatorname{ind}_{T_{f}} g: \zeta_{f}^{0}=\zeta_{f} \text { on } T_{f}^{(o)}, g \in S_{f}\right\} \\
= & \operatorname{lcm}\left\{\operatorname{ind}_{T} x: \zeta^{[x]}=\zeta \text { on } K\left(T^{(x)}\right)_{f}, x \in S\right\},
\end{aligned}
$$

where $\zeta^{[x]}([t])=\zeta\left([x][t][x]^{-1}\right)$ for $t \in T^{(x)}$ defines $\zeta^{[x]}$ on $K\left(T^{(x)}\right)_{f}$.

The above development proves the following analogue to the last part of Theorem A for twisted group algebras:

THEOREM B. Let $S$ be a group with factor set $f$ and $T$ a subgroup of $S$. Assume that $f$ has values which are powers of a fixed primitive nth root of unity in $K$ and that [1] is the identity for $K(S)_{f}$. If $\chi$ is an irreducible character of $K(S)_{f}$ and $\zeta$ is a linear character of $K(T)_{f}$ such that $\zeta$ appears in the restriction of $\chi$ to $K(T)_{f}$, then the degree $\chi([1])$ of $\chi$ divides

$$
[S: T] \operatorname{lcm}\left\{\operatorname{ind}_{T} x: \zeta^{[x]}=\zeta \text { on } K\left(T^{(x)}\right)_{f}, x \in S\right\},
$$

where $\zeta^{[x]}([t])=\zeta\left([x][t][x]^{-1}\right)$ for all $t \in T^{(x)}$.

2. The two-step case. Let $G$ be a group, $N$ a normal subgroup of $G$, and suppose $H$ is a subgroup of $G$ such that $N \subseteq H \subseteq G$. Let $\psi, \zeta, \chi$ be irreducible characters of $N, H, G$, respectively, such that they satisfy the following compatibility conditions:

$$
\psi(1)=1, \quad\left(\psi^{H}, \zeta\right)=1, \quad\left(\zeta^{G}, \chi\right) \geqq 1 .
$$

Let $e$ be the idempotent in $K N$ such that $K N e$ affords $\psi$, namely $e=$ $|N|^{-1} \sum_{n \in N} \psi\left(n^{-1}\right) n$, and let $E=e K G e$. Set $S=\left\{g \in G: \psi^{g}=\psi\right.$ on $\left.N\right\}$; observe that $N^{(g)}=N$ for all $g \in G$ since $N$ is normal in $G$, and $S$ is a subgroup of $G$ containing $N$. Write $S=U g_{i} N$ (disjoint). By Theorem A, $E=e K G e$ has a basis consisting of $\left\{e g_{i} e\right\}$. Note that for $g \in S$, $e g e=e g=g e$. Further, in writing $S=\cup_{g_{i}} N$ (disjoint) we assume that $g_{1}=1$, and we fix this coset decomposition of $S$ in what follows.

To multiply $g_{i} e$ and $g_{j} e$ in $E$ we write $g_{i} g_{j}=g_{k} n$ for some $n \in N$; then

$$
\left(g_{i} e\right)\left(g_{j} e\right)=\left(g_{i} g_{j}\right) e^{2}=g_{k} n e=\psi(n)\left(g_{k} e\right) .
$$

Let $\bar{S}=S / N$; then $\bar{S}$ is the set of cosets $\left\{g_{i} N\right\}$ given above. Define a factor set $f$ on $\bar{S} \times \bar{S}$ via $f\left(g_{i} N, g_{j} N\right)=\psi(n)$ where $g_{i} g_{j}=g_{k} n, n \in N$. From these remarks we have identified $E$ with $K(\bar{S})_{f}$ via $g_{i} e \leftrightarrow\left[g_{i} N\right]$. 
Similarly let $F=e K H e$ and $T=S \cap H$. Then $F$ may be identified with $K(\bar{T})_{f}$, where $\bar{T}=T / N$ and $\bar{T}$ is a subgroup of $\bar{S}$.

By the compatibility conditions $(*), \zeta$ and $\chi$ are irreducible characters of $K H$ and $K G$, respectively, such that $\left(\psi^{H}, \zeta\right)>0$ and $\left(\psi^{G}, \chi\right)$ $>0$. By Theorem A, the restrictions $\zeta_{F}$ of $\zeta$ to $F$ and $\chi_{E}$ of $\chi$ to $E$ are irreducible characters of $F$ and $E$, respectively. Moreover, $\left(\psi^{H}, \zeta\right)=1$ implies that $\zeta_{F}$ is a linear character of $F$, and $\left(\zeta^{G}, \chi\right) \geqq 1$ implies that the restriction $\chi_{F}$ of $\chi_{E}$ to $F$ contains $\zeta_{F}$ with positive multiplicity. By Theorem $\mathrm{B}$ (observing that $[\overline{1}]$ is the identity for $E$, where $\overline{1}$ $=1 \cdot N=N \in \bar{S})$ the degree $\chi_{E}([\overline{1}])$ of $\chi_{E}$ divides

$$
[\bar{S}: \bar{T}] \operatorname{lcm}\left\{\operatorname{ind}_{\bar{T}} \sigma: \zeta^{[\sigma]}=\zeta \text { on } K\left(\bar{T}^{(\sigma)}\right)_{f}, \sigma \in \bar{S}\right\},
$$

where $\zeta^{[\sigma]}([\tau])=\zeta\left([\sigma][\tau][\sigma]^{-1}\right)$ for all $\tau \in \bar{T}$.

By Theorem A we may write the block idempotents in $E$ and $F$ corresponding to $\chi_{E}$ and $\zeta_{F}$, respectively, as follows:

$$
\begin{aligned}
& e_{1}=[G: N]^{-1} \chi(1) \sum_{\sigma \in \bar{S}} \chi\left([\sigma]^{-1}\right)[\sigma] \in E \\
& e_{2}=[H: N]^{-1} \zeta(1) \sum_{\tau \in \bar{T}} \zeta\left([\tau]^{-1}\right)[\tau] \in F .
\end{aligned}
$$

Note that $N$ is normal in $G$ so the terms $\operatorname{ind}_{N} g$ are all one. Also, in the identification of $e g_{i} e$ with $\left[g_{i} N\right]$ we see that $e g_{i}^{-1} e$ corresponds to $\left[g_{i} N\right]^{-1}$.

By the development of $\$ 1$, we may calculate the block idempotents $e_{1}$ and $e_{2}$ in $K(\bar{S})_{f}$ and $K(\bar{T})_{f}$ in terms of the irreducible characters $\chi_{E}$ and $\zeta_{F}$. Indeed,

$$
\begin{aligned}
& e_{1}=|\bar{S}|^{-1} \chi([\overline{1}]) \sum_{\sigma \in \bar{S}} \chi\left([\sigma]^{-1}\right)[\sigma] \\
& e_{2}=|\bar{T}|^{-1} \zeta([\overline{1}]) \sum_{\tau \in \bar{T}} \zeta\left([\tau]^{-1}\right)[\tau] .
\end{aligned}
$$

Comparing the coefficients of $[\overline{1}]$ in (2) and (3) we obtain

$$
\begin{aligned}
& {[G: N]^{-1} \chi(1) \chi([\overline{1}])=|\bar{S}|^{-1} \chi([\overline{1}])^{2}} \\
& {[H: N]^{-1} \zeta(1) \zeta([\overline{1}])=|\bar{T}|^{-1} \zeta([\overline{1}])^{2} .}
\end{aligned}
$$

But $\zeta_{F}$ is linear on $F$ so $\zeta([\overline{1}])=1$. Therefore the above become

$$
\begin{aligned}
& {[G: N]^{-1} \chi(1)=|\bar{S}|^{-1} \chi([\overline{1}])} \\
& {[H: N]^{-1} \zeta(1)=|\bar{T}|^{-1} .}
\end{aligned}
$$

Let $l=\operatorname{lcm}\left\{\operatorname{ind}_{\bar{T}} \sigma: \zeta^{[\sigma]}=\zeta\right.$ on $\left.K\left(\bar{T}^{(\sigma)}\right)_{f}, \sigma \in \bar{S}\right\}$. Then (1) above becomes $[\bar{S}: \bar{T}] l$. But $|\bar{S}|=[S: N],|\bar{T}|=[T: N]$ and $N \subseteq T \subseteq S$, so 
that $[\bar{S}: \bar{T}]=[S: T]$. Therefore $\chi([\overline{1}])$ divides $[S: T] l$.

By (5) this is just restating that $\chi(1)$ divides $[S: N]^{-1}[G: N][S: T] l$ which equals $[T: N]^{-1}[G: N] l$. But by the second equation of $(5)$ this is just $\zeta(1)[G: H] l$. Therefore

$$
\chi(1) \text { divides } \zeta(1)[G: H] l .
$$

As in $\$ 1$, one checks that

$$
\begin{aligned}
l & =\operatorname{lcm}\left\{\operatorname{ind}_{\bar{T}} \sigma: \zeta^{[\sigma]}=\zeta \text { on } K\left(\bar{T}^{(\sigma)}\right)_{f}, \sigma \in \bar{S}\right\} \\
& =\operatorname{lcm}\left\{\operatorname{ind}_{T} x: \zeta^{x}=\zeta \text { on } T^{(x)} e, x \in S\right\},
\end{aligned}
$$

where $T^{(x)} e=\left\{t e: t \in T^{(x)}\right\}$. We then obtain the following result:

Theorem C. Let $G$ be a finite group, $N$ a normal subgroup of $G$, and let $H$ be a subgroup of $G$ such that $N \subseteq H \subseteq G$. Let $\psi, \zeta, \chi$, be irreducible characters of $N, H, G$, respectively, satisfying the compatibility conditions (*). If $S=\left\{g \in G: \psi^{\circ}=\psi\right.$ on $\left.N\right\}$ and $T=H \cap S$ then the degree $\chi(1)$ of $\chi$ divides

$$
[G: H] \zeta(1) \operatorname{lcm}\left\{\operatorname{ind}_{T} x: \zeta^{x}=\zeta \text { on } T^{(x)} e, x \in S\right\},
$$

where $T^{(x)} e=\left\{t e: t \in T^{(x)}\right\}$ and $e=|N|^{-1} \sum_{n \in N} \psi\left(n^{-1}\right) n$.

REMARK. Let $H$ be a subgroup of the group $G, \zeta$ a linear character of $H$ and $\chi$ an irreducible character of $G$ such that $\left(\zeta^{G}, \chi\right) \geqq 1$. Let $N=\{1\}$ and $\psi=1$, the identity character on $N$. Then $\left(\psi^{H}, \zeta\right)=\left(\psi, \zeta_{N}\right)$ $=1$, so the hypotheses of Theorem C are satisfied. Clearly $S=G$, $T=H$, and $e=1$ as in Theorem $\mathrm{C}$. Thus the conclusion of the theorem reads "the degree $\chi(1)$ of $\chi$ divides $[G: H] \operatorname{lcm}\left\{\operatorname{ind}_{H} x: \zeta x=\zeta\right.$ on $H^{(x)}$, $x \in G\}$," which is exactly the last part of Theorem A.

Note also that the theorem is a triviality if $\zeta^{G}=\chi$, for then $\chi(1)$ $=[G: H] \zeta(1)$ automatically.

Corollary. Hypotheses as in Theorem $\mathrm{C}$. If in addition $H$ is normal in $G$, then the degree $\chi(1)$ of $\chi$ divides $[G: H] \zeta(1)$.

Proof. Since $H$ is normal in $G, T$ is normal in $S$, and so ind $_{T} x=1$ for all $x \in S$. This proves the corollary.

Let $G$ be a nonabelian group of order six, $H$ a subgroup of order two. If $\chi$ is the character of degree two on $G$, then $\chi$ appears with positive multiplicity in a linear character $\zeta$ on $H$. But clearly in this case $\chi(1)$ does not divide $[G: H] \zeta(1)$. 
It would be delightful to know if one could somehow drop the subgroup $N$ and the linear character $\psi$ from the hypotheses of Theorem $C$ and yet obtain a similar result about the degree of $\chi$.

\section{REFERENCES}

1. C. W. Curtis and T. V. Fossum, On centralizer rings and characters of representations of finite groups, Math. Z. 107 (1968), 402-406.

2. C. W. Curtis and I. Reiner, Representation theory of finite groups and associative algebras, Pure and Appl. Math., vol. 11, Interscience, New York, 1962. MR 26 \#2519.

UNIVERSITY OF UTAH 